\title{
An Insight into Pathogenesis of Cardiovascular Diseases
}

\section{Rakhshinda Zafar}

117 country club lane, Kittanning PA 16201, USA

*Corresponding author: Rakhshinda Zafar, 117 country club lane, Kittanning PA 16201, USA. Tel: 724-525-5981; E-mail: docrzafar@hotmail.com

Received date: March 31, 2015; Accepted date: April 24, 2015; Published date: April 27, 2015

Copyright: (c) 2015 Zafar R. This is an open-access article distributed under the terms of the Creative Commons Attribution License, which permits unrestricted use, distribution, and reproduction in any medium, provided the original author and source are credited.

\begin{abstract}
Based on my clinical experience and knowledge gained through extensive research in this area, I have come up with a hypothesis which sheds more light in the pathogenesis of cardiovascular diseases. Atherosclerosis is implicated as playing the key role in the pathogenesis of cardiovascular diseases which involves large and medium sized arteries. There is now evidence that atherosclerosis is an immuno- inflammatory process.

My hypothesis is that chronic psychosocial stress is the main trigger for the systemic inflammation which results by activation of hypothalamus-pituitary-adrenal (HPA) axis and sympathetic-adrenal-medullary (SAM) axis. This stress response sets into motion innate immune response, initiating a cascade of events which include: release of neuro-endocrine transmitters, endothelial dysfunction, increased permeability of micro vascular circulation and increased delivery of free fatty acids in circulation among others. Liver responds by increased low density lipoproteins production which continuously enter the arteries, excess LDL is transformed into oxidized low density lipoproteins (ox LDL). ox LDL goes through pattern recognition and is recognized as antigen. Adaptive immunity is activated. ox LDL is pro oxidant, it results in inflammation, reactive oxygen species are released causing oxidative stress. This causes atherosclerosis in large and medium sized arteries and tissue damage in organs sub served by micro vasculature. Hence the process being systemic inflammation is not limited to large and medium sized arteries but is global in reach involving the entire vasculature.
\end{abstract}

This article will review stress and pathophysiology of stress, pathophysiology of cardiovascular diseases which includes pathogenesis of atherosclerosis and Inflammation in atherosclerosis. It discusses microcirculation, and goes over disorders involving microcirculation. Oxidative stress is reviewed followed by conclusion.

\begin{abstract}
Abbreviations:
Hypothalamic-pituitory-adrenal (HPA); sympathetic-adrenalmedulla (SAM); central nervous system (CNS); brain neurotropic factor (BNDF); polysciated Neural Cell adhesion molecule (PSANCAM); tissue plasminogen activator (Tpa); nuclear factor $\mathrm{Kb}$ (NFKB); cortico trophin releasing factor (CRF); adrenocorticotrophin hormone (ACTH); Interleukin -6 (IL_6); acute phase response (APR); nitric oxide (NO); reactive oxygen species (ROS); E nitrogen oxide synthase (Enos); high density lipoprotein (HDL); low density lipoprotein (LDL); cardiovascular disease (CVD); coronary heart disease (CHD); ankle brachial index (ABI); peripheral arterial disease (PAD); renal artery stenosis (RAS); A atherosclerotic cardiovascular disease (ASCVD); blood pressure (BP); diabetes mellitus (DM); cardiovascular $(\mathrm{CV})$; $\mathrm{C}$ reactive protein $(\mathrm{CRP})$; endothelial cells (ECS); metabolic syndrome (Met S); chronic kidney disease (CKD); chronic obstructive pulmonary disease (COPD); non-alcoholic fatty liver disease (NAFLD); reactive nitrogen species (RNS); atrial fibrillation(AF); oxidized low density lipoprotein (Oxldl); coronary artery calcium (CAC); carotid intima media thickness (CIMT); muscle sympathetic nerve activity (MSNA)
\end{abstract}

\section{Review}

This article will review stress and pathophysiology of stress, pathophysiology of cardiovascular diseases which includes pathogenesis of atherosclerosis and inflammation in atherosclerosis. It discusses microcirculation, and goes over disorders involving microcirculation. Oxidative Stress is reviewed followed by conclusion.

\section{Stress}

The term stress has changed its meaning from that of a nonspecific body response to a 'monitoring system of internal and external cues' that is a modality of reaction of the mammalian central nervous system (CNS) which is critical to the adaptation of the organism to its environment [1]. Other working definition of stress is a physiological response that serves as a mechanism of mediation on linking any given stressor to its target organ effect. Stress begins in the brain and affects the brain as well as rest of the body [2].

\section{Stress response}

Most stressors are psychosocial and they set the stage for stress response. Cognitive appraisal mechanism determines affect (felt emotion). Once appraisal is made, efferent impulses project to highly sensitive emotional anatomy in the limbic system especially in hippocampus to trigger visceral effector mechanism. Impulses also project to areas of neocortex concerned with neuromuscular behavior.

Stress hormones and other mediators such as nuero transmitters, cytokines and other hormones are essential for adaptation to challenges of daily life as well as to major life stressors. This process has been called "allostasis" that is maintaining stability or homeostasis through change. When mediators of allostasis like cortisol and adrenaline are released in response to stressor or lifestyle factors such 


\section{$10.4172 / 2329-9517.1000197$}

Page 2 of 7

as diet, sleep and exercise they promote adaptation and survival and are generally beneficial.

Chronic stress can promote and exacerbate pathophysiology through the same systems that are dysregulated. The burden of chronic stress and accompanying changes in personal behaviors smoking, eating too much, drinking, poor quality sleep otherwise referred to as "lifestyle" is called allostatic load.

Brain region such as hippocampus, prefrontal cortex and amygdalla respond to acute and chronic stress. The adaptive plasticity of chronic stress involves many mediators including glucocorticoids, excitatory amino acids, endogenous factors such as brain neurotropic factor (BNDF), polysciated neural cell adhesion molecules (PSA-NCAM) and tissue plasminogen activator (tPA) [3].

In response to a stressor physiological changes are set into motion to help the individual cope with the stressor. However chronic activation of the stress response which includes the hypothalamus pituitary-adrenal (HPA) axis and the sympathetic-adrenal medullary (SAM) axis results in chronic production of glucocorticoids hormones and catecholamines.

Glucocorticoid receptors exposed on a variety of immune cells bind cortisol and interfere with the function of NF $\mathrm{kB}$ which regulates the activity of cytokine producing immune cells.

Adrenergic receptors bind epinephrine and nor-epinephrine and activate the cAMP response element binding protein, inducing the transcription of genes encoding for a variety of cytokines.

The changes in gene expression mediated by glucocorticoids and catecholamines can dysregulate immune function. The magnitude of stress associated immune dysregulation is large enough to have health implications [4].

There are several articles which demonstrate the role of stress in various disease processes. The article published in Annals of The New York Academy of Sciences describes that stress promotes adaptation, but prolonged stress leads over time to wear- and- tear on the body (allostatic load). Neural changes mirror the pattern seen in other body systems, that is, short term adaptation vs. long term damage. Allostatic load leads to impaired immunity, atherosclerosis, obesity, bone demineralization and atrophy of nerve cells in the brain. Many of these processes are seen in major depressive illnesses and may be expressed in other chronic anxiety disorders [5].

The study published in American Journal of Psychiatry describes that significant life stress including early life stress a major risk factor for development of major depression, once manifested major depression has been associated with enhanced tonic activation of the innate immunity system including increased plasma pro inflammatory cytokines such as interleukin (IL) [6]. In non-depressed individuals exposure to psychosocial stress has been associated with increased plasma IL - 6 response as well as activation of nuclear factor (NF) $-k B$, a transcription factor that serves as lynchpin in the inflammatory signal cascade.

The study findings suggested that male major depression patients with early life stress may clinically exhibit stress induced increases in inflammatory markers that are further exacerbated by exposure to acute stress. These findings indicate that depressed patients with increased early life stress exhibit both a baseline hyper inflammatory state coupled with a hyper responsive inflammatory response to stress which together may contribute to medical co morbidities associated with major depression and inflammation, such as cardiovascular diseases.

Previous stress exposure has been shown to sensitize subsequent immune responses to immune challenge and therefore the findings may result from an interaction between major depression and early life stress.

Sympathetic nervous system activation has been shown to enhance inflammatory responses and major depression, patients with early life stress has been shown to exhibit enhanced sympathetic nervous system response to stress challenge [6].

The article published in Brain, Behavior and Immunity describes the relationship of stress and the inflammatory response. The author reviews the subject of neuro inflammation. In response to psychological stress or certain physical stressors an inflammatory process may occur by release of neuropeptides especially substance $P$ (SP) or other inflammatory mediators from sensory nerves and the activation of mast cells or other inflammatory cells. Central neuropeptides particularly corticosteroids releasing factor (CRF) and perhaps SP as well, initiate a systemic response by activation of neuro endocrinological pathways such as the sympathetic nervous system, hypothalamus pituitary axis and the renin angiotensin system with the release of stress hormones (i.e, catecholamines, corticosteroids, growth hormones, glucagon and renin). These together with cytokines induced by stress initiate acute phase response (APR) and the induction of acute phase proteins, essential mediators of inflammation. Central nervous system nor-epinephrine may also induce the APR perhaps by macrophage activation as may lipopolysaccharide which the author postulates induces cytokines from hepatic Kupffer cells subsequent to an enhanced absorption from the gastrointestinal tract during psychologic stress. The brain may initiate or inhibit the inflammatory process. The inflammatory response is contained within the psychosocial stress response which evolved later. Moreover the same neuropeptides (CRF and possibly SP as well) mediate both stress and inflammation. Cytokines evoked by either stress or inflammation response may utilize similar somatosensory pathways to signal the brain, other instances whereby stress may induce inflammatory changes are reviewed in the article. The author postulates that repeated episodes of acute or chronic psychogenic stress may result in atherosclerosis in the arteries or chronic inflammatory changes in other organs as well [7].

\section{Pathogenesis of Atherosclerosis}

Atherosclerosis is a multifocal, smoldering immuno-inflammatory disease of medium-sized and large arteries fuelled by lipids. Endothelial cells, leukocytes and intimal smooth muscle cells are the major players in the development of disease.

\section{Atherogenic stimuli}

Among many cardiovascular risk factors, elevated plasma cholesterol is unique in being sufficient to drive development of atherosclerosis. Other risk factors such as hypertension, diabetes, smoking, male gender and possibly inflammatory markers e.g Creactive protein, cytokines and so on appear to accelerate a disease driven by atherogenic lipoproteins, the first of which being LDL. The importance of risk factors beyond cholesterol is documented by great disparity in the expression of clinical disease. 


\section{Cellular component of atherosclerosis}

\section{Endothelial cells}

Atherosclerotic lesion begins to develop under an intact but leaky, activated and dysfunctional epithelium. Later endothelial cells may vanish and de- endothelialized (denuded) areas appear with or without platelets adhering to the exposed sub endothelial tissue.

Depending on size and concentration, plasma molecules and lipoprotein particles extravasate through the leaky and defective endothelium into the subendothelial space, where potentially atherogenic lipoproteins are retained and modified (oxidized) and become cytotoxic, pro inflammatory, chemotactic and pro atherogenic. The mechanism for this modification is unknown but could be oxidation mediated by myeloperoxidases, lipooxygenase, and /or nitric oxide synthase (NOS).

Nitric oxide is a potent oxidant produced by both endothelial cells and macrophages that appear to exert both protective and atherogenic effect. NO produced by NOS in macrophages is pro atherogenic.

The endothelium becomes activated by atherogenic and pro inflammatory stimuli and the expression of adhesion molecules, primarily vascular cell adhesion molecule -1 (VCAM-1), are up regulated and monocytes and $\mathrm{T}$ cells are recruited. Other adhesion molecules intracellular adhesion molecule - 1, E Selectin and P Selectin probably contribute to recruitment of blood borne cells to the atherosclerotic lesions.

Endothelial dysfunction as assessed clinically (impaired nitric oxide mediated vasodilatation) predicts clinical events caused by atherosclerosis. It is indeed thought provoking that mere presence of risk factors is associated with endothelial dysfunction not only in atherosclerosis susceptible arteries but also in arteries that are relatively resistant to atherosclerosis e.g. the brachial artery.

\section{Leukocytes}

One of the earlier responses in atherogenesis is focal recruitment of circulating monocytes and to lesser extent $\mathrm{T}$ lymphocytes. The persistence of this cellular response seems to underlie disease progression. B lymphocytes and plasma cells are rare in intimal plaque but may be abundantly present in adventitia next to advanced intimal disease. Activated mast cells may be found both in plaque and adventitia, particularly in culprit lesions causing acute ischemic events. Neutrophils are rare in the complicated atherosclerosis.

Mere adhesion to the endothelium of blood borne cells is not enough to arrive in the lesions, trans endothelial migration is required. One or more chemokines are necessary. Endothelial cells, smooth muscle cells and macrophages all contribute to over expression of MCP-1 (Monocyte chemotactic protein).

Within the intima monocytes differentiate into macrophages and internalize atherogenic lipoproteins via so called scavenger receptors. The development of lipid loaded macrophages containing massive amounts of cholesterol esters (foam cells) is a hallmark of both early and late atherosclerotic lesions. With continuing supply of lipoproteins, the macrophages eat until they die, in contrast to native LDL receptor, scavenger receptors are not down regulated by cellular cholesterol accumulation.

The death of macrophages by apoptosis and necrosis contributes to soft and destabilizing lipid rich core within the plaque. On the other hand macrophages may under appropriate conditions (low LDL and high HDL) shrink by effluxing cellular cholesterol to extra cellular HDL via membrane transporter, the initial step in "reverse cholesterol transport". Aside from scavenger function macrophages also possess destabilizing and thrombotic properties by expressing matrix metalloproteinases and tissue factor [8].

\section{Innate immunity in atherosclerosis}

Early involvement of monocytes/ macrophages is the most prominent cellular component of the innate immunity response during atherogenesis. The recruitment of mononuclear phagocytes involves attachment to activated endothelial cells by the leukocyte adhesion molecules, several protein mediators, specialized cytokines known as chemokines, direct cell migration of monocytes into the intima. Maturation of monocytes into macrophages their multiplication and production of many mediators ensues.

Monocytes entry occurs not just during the initial stages but continues even in established lesions.

Hyperlipidemia elicits a profound enrichment of pro-inflammatory subset of monocytes in the mouse. These pro inflammatory monocytes home to atherosclerotic lesions, where they propogate the innate immune response by expressing high levels of pro inflammatory cytokines and other macrophage mediators including matrix metalloproteinases.

Recent evidence has also highlighted the potential participation of mast cells in atherosclerosis.

Many links exist between lipoproteins and innate immunity. Modified lipoproteins interact with scavengers receptors and may thus send pro- inflammatory signals and oxidized phospholipids derived from modified lipoproteins may also drive inflammation.

There is also link between thrombosis and inflammation. A major protein mediator of coagulation, thrombosis can elicit the expression of pro inflammatory cytokines from vascular endothelium and smooth muscle cells. Platelets when activated can secrete preformed pro inflammatory cytokines and exteriorize and shed multi-potent pro inflammatory stimulus CD 40- ligand. Platelets can also release a pro inflammatory mediator known as (MRP-8/14. MRP- 8/14 can bind TLR4 activating innate immunity through this pattern recognition receptor. This ligand can also promote endothelial cell apoptosis a process implicated in plaque thrombosis. These observations tighten the link between inflammation and thrombosis suggesting an intimate interlacing of these 2 convergent pathways in atherosclerosis.

\section{Adaptive immunity}

Accumulating evidence suggests a key regulating role of adaptive immunity in atherosclerosis and its complications. Interacting with a special subset of mononuclear phagocytes specialized in antigen presentation known as dendritic cells.

$\mathrm{T}$ lymphocytes encounter antigens and mount a cellular immune response. The dendritic cells populate atherosclerotic plaques and regional draining lymph nodes, where they can present antigens to $\mathrm{T}$ cells with co -stimulating molecules that incite this key afferent nodes for adaptive immunity. Putative antigens that stimulate $T$ cells in the context of atherosclerosis include certain heat shock proteins, components of plasma lipoproteins and potentially microbial structures as well. The clone of $\mathrm{T}$ cells that recognizes $\mathrm{T}$ cells in this 


\section{(10.4172/2329-9517.1000197}

Page 4 of 7

context will proliferate to amplify the immune response. Upon renewed exposure to the specific antigen these $\mathrm{T}$ cells produce cytokines and trigger inflammation and some $\mathrm{T}$ cells have mechanisms specialized for killing cells. This amplification accounts for the delay in the typical adaptive immune response that is slower and much more structurally specific than "fast and blunt "innate immune response [9].

\section{Cardiovascular diseases}

Cardiovascular diseases develop as a result of atherosclerosis involving large and medium sized arteries such as diseases of coronary arteries, diseases of aorta, diseases of carotid arteries and peripheral arteries which include visceral arteries, renal arteries, and upper and lower extremity arteries.

A host of risk factors are associated with these conditions such as hypertension, dyslipidemias, diabetes- mellitus, ageing, cigarette smoking, obesity, metabolic syndrome and newly emerging risk factors i.e. hs CRP, Carotid intima media thickness (CIMT), Coronary artery calcium score (CAC) and Ankle brachial index (ABI).

Other risk factors are atherogenic diet, physical inactivity, positive family history of premature coronary artery disease, and male gender.

Cardiovascular diseases can be categorized as macro vascular diseases.

\section{C-Reactive protein}

$\mathrm{C}$ - Reactive protein (CRP) is a $110 \mathrm{kDa}$ protein predominantly synthesized by hepatocytes in response to the inflammatory cytokines, interleukin (IL)-6 and IL - 1 .

CRP was the first acute phase protein to be described and is an exquisitely sensitive marker of inflammation and tissue damage. The acute phase response comprises the non-specific physiological and biochemical responses of endothermic animals to most forms of tissue damage, infection, inflammation and malignant neoplasia. In particular, the synthesis of a number of proteins is rapidly up regulated, principally in hepatocytes under the control of cytokines originally at site of pathology [10].

\section{Discussion}

After reviewing the knowledge gained by pathophysiology of chronic psychosocial stress and atherosclerosis, it appears that atherosclerosis is driven by activation of innate immune response, triggered by activation of HPA axis and SAM axis. One of the processes initiated is mobilization of free fatty acids in circulation. Liver responds by producing LDL-C, which continuously reaches the arteries and is converted into oxLDL which initiates inflammation i.e. atherosclerosis, resulting in cardiovascular diseases.

\section{Microcirculation}

The microcirculation is highly responsive to and a vital participant in the inflammatory response. All the segments of the microcirculature (arterioles, capillaries and venules) exhibit characteristic phenotypic changes during inflammation that appear to be directed toward enhancing the delivery of inflammatory cells to the injured/infected tissue, isolating the region from healthy tissue and the systemic circulation and setting the stage for tissue repair and regeneration. The best characterized responses of the microcirculation to inflammation include impaired vasomotor function, reduced capillary permeability, and an increase in the rate of proliferation of blood and lymphatic vessels. A variety of cells that normally circulate in blood i.e. leukocytes, platelets or reside within the vessel wall (endothelial cells, pericytes) or in the perivascular space (mast cells), macrophages) are activated in response to inflammation. The activation products and chemical mediators released from the cells act through different wellcharacterized signaling pathways to induce the phenotypic changes in micro vessel function that accompany inflammation [11].

I will now describe some of the diseases which are caused as a result of systemic inflammation involving microcirculation.

\section{Hypertension}

\section{The role of oxidative stress}

Primary hypertension/essential hypertension accounts for $90 \%$ of all cases of hypertension. Increased sympathetic activity contributes due to activation of stress response among other factors. Oxidative stress results of excessive generation of reactive oxygen species (ROS) has a key role in the pathogenesis of hypertension. The modulation of the vasomotor system initiates ROS as mediators of vasoconstriction induced by angiotensin 11, endothelin 1 and urotensin 11 among others. Bioavailability of NO major vasodilator is highly dependent in the redox state.

Under physiological condition low concentration of intracellular ROS have an important role in the redox signaling monitoring vascular function and integrity.

Under pathological conditions increased level of ROS contributes to vascular dysfunction and remodeling through oxidative damage. In human hypertension increase in the production of superoxide anions and hydrogen peroxide, a decrease in NO synthase and a reduction in antioxidant availability have been observed [12].

\section{Diabetes mellitus}

The current literature supports the role of oxidative stress in vascular disease in diabetes.

The Diabetes is associated with macro vascular manifestations which include atherosclerosis and medial calcification. The micro vascular consequences, retinopathy and nephropathy are major causes of blindness and end stage renal disease. The pathophysiology of vascular disease in diabetes involves abnormalities in endothelial, vascular smooth muscle cells and platelet function. The metabolic abnormalities, hyperglycemia, increased free fatty acids and insulin resistance each provoke mechanisms that contribute to vascular dysfunction. These include decreased bioavailability of NO, increased oxidative stress, disturbance of intracellular signal transduction and activation of receptors for AGEs. In addition platelet function is abnormal and there is increased production of several pro thrombotic factors. The abnormalities contribute to the cellular events that cause atherosclerosis and increased risk of cardiovascular disease events that occur in patients with diabetes and atherosclerosis [13].

There is increasing evidence that an ongoing cytokines-induced acute phase response (sometimes called low grade inflammation), but part of wide spread activation of the innate immune system) is closely involved in the pathogenesis of type 2 diabetes and associated complications such as dyslipidemia and atherosclerosis. Elevated circulating inflammatory markers such as c - Reactive protein and interleukin - 6 predict the development of type 2 diabetes and several 


\section{(10.4172/2329-9517.1000197}

Page 5 of 7

anti-inflammatory proteins lower both acute phase reactions and glycemia. Aspirin and Thiazolidinediones possibly decreases the risk of developing type 2 diabetes (statins). Among the risk factors for type-2 diabetes, which are also known to be associated with activated innate immunity are age, inactivity, certain dietary components, smoking psychosocial stress and low birth weight. Activated immunity may be the common antecedent of both type 2 diabetes and atherosclerosis, which probably develop in parallel. Other features of type 2 Diabetes such as fatigue, sleep disturbance and depression are likely to be at least partly due to hypercytokinemia and activated innate immunity.

There has been a recent explosion of interest in the notion that chronic low grade infection and activation of the innate immune system are closely involved in the pathogenesis of type 2 Diabetes. For example since this hypothesis was first proposed in 1997 and 1998 at least 12 studies have shown that circulating markers of inflammation acute phase reactants or interleukin -6 (IL)-6, (the major cytokine mediator of the acute phase response) are strong predictors of the development of type 2 diabetes [14].

\section{Ageing}

Ageing has come out as an important risk factor for CVD. As reviewed in earlier section ageing phenomenon is the result of mild chronic allostatic load and is due to micro vascular circulation disease.

The article published in American physiological society states, "You're as old as your arteries" describes, endothelial dysfunction develops with age increases the risk of age associated vascular disorders. Nitric oxide insufficiency, oxidative stress and chronic low grade inflammation induced by up regulation of adverse cellular signaling processes and imbalances in stress resistance pathways, mediate endothelial dysfunction with ageing. Healthy life style behaviors preserve endothelial function with ageing by inhibiting these mechanisms [15].

\section{Metabolic syndrome}

High level of free radicles together with low anti-oxidant capacity detected in obese adults indicate elevated oxidative stress which is together with systemic inflammation - further potentiated in the case of obese patients with metabolic syndrome. This imbalance in oxidative /anti oxidative state and subclinical inflammatory state leads to higher risk of atherosclerosis and diabetic complications [16].

\section{Chronic kidney disease}

\section{Sympathetic hyperactivity in CKD}

Cardiovascular morbidity and mortality importantly influence life expectancy of patients with CKD. Traditional risk factors are usually present but several other factors have been identified. CKD is often characterized by an activated sympathetic -renin system. This may contribute to pathogenesis of renal hypertension but it may affect adversely prognosis independent of its effect on blood pressure.

Hypertension is common in patients with CKD. Prevalence varies between $30-100 \%$ depending on target population, cause of renal disease and level of renal function. Hypertension has been viewed as volume dependent. CKD is often characterized by an activated sympathetic nervous system.
Sympathetic nervous system is a part of autonomic nervous system. Its activity can be derived indirectly from sympathetic effector response i.e. blood pressure or heart rate. Nor-epinephrine in plasma is net result of discharge, reuptake, metabolism and clearance, not suitable for accurate measure of the disease. Sleep apnea associated with sympathetic hyperactivity. LVH is related to increased sympathetic activity. Increased sympathetic activity is associated with Congestive heart failure, arrhythmias and atherosclerosis in experimental animals [17].

\section{Role of oxidative stress and antioxidant therapy}

Oxidative stress is frequently associated with and is partly involved in the pathogenesis of chronic renal failure, hypertension and their complications.

The up regulation of nicotinamide adenosine dinucleotide phosphate (reduced form) oxidase and tubulo-interstitial accumulation of activated $\mathrm{T}$ cells, macrophages and superoxide producing cells are partly responsible for oxidative stress in several models of hypertension. Antioxidant therapy alleviates hypertension, averts nuclear factor kappa $B$ activation and mitigates tubulointerstitial inflammation in hypertensive animals.

Oxidative stress contributes to hypertension, endothelial dysfunction and brain disorder in chronic renal failure and down regulation of superoxide dismutase and up regulation of nicotinamide adenosine dinucleotide phosphate (reduced form) [18].

\section{Psychiatric disorders}

Depression, chronic anxiety disorders and schizophrenia. Increasingly physical disorders such as obesity, hypertension and type 2 diabetes are being recognized as significant co morbidities in people with severe mental illnesses, including psychotic disorders such as Schizophrenia. Whether these disorders are part of the disease process itself through increased stress and inflammatory responses, genetic vulnerabilities or environmental factors versus sequel of the treatment of the disease, has been a matter of debate.

Recent evidence has suggested that HPA axis- dysregulation - also may play a significant role in the development of various components of the Met S. While increased cortisol production is a normal response in acute stress, several studies have demonstrated a disruption in normal HPA axis activity and relative hypercortisolemia in patients with Schizophrenia. Ryan, et al. have recently shown that first- episode treatment - naïve patients with Schizophrenia demonstrated a significantly higher plasma cortisol level along with higher percentage of patients having impaired fasting glucose, increased fasting glucose and increased insulin resistance compared with matched control group [19].

Clinical depression and influencing risk markers of coronary heart disease. The study examined the relation between depression and the expression of inflammatory risk markers implicated in the pathogenesis of coronary heart disease. One hundred adults were enrolled. Fifty subjects met the diagnostic criteria for clinical depression. The remaining were demographically matched controls with no history of psychiatric illness. The depressed subjects exhibited significantly higher levels of the inflammatory marker C-reactive protein and interleukin -6 compared with control subjects. These findings indicate that in otherwise healthy adults depression is 


\section{(10.4172/2329-9517.1000197}

Page 6 of 7

associated with heightened expression of inflammatory markers implicated in pathogenesis of Coronary Heart Disease [20].

\section{Non-alcoholic fatty liver disease}

Although the epidemic of obesity has been accompanied by an increase in the prevalence of the metabolic syndrome, not all obese develop the syndrome even lean individuals can be insulin resistant. Both lean and obese insulin resistant individuals have an excess of fat in the liver which is not attributable to alcohol or other known causes of liver disease, a condition defined as non-alcoholic fatty liver disease (NAFLD) by gastroenterologist. The fatty liver is insulin resistant. Liver fat is highly significantly and linearly correlated with all components of the metabolic syndrome independent of obesity, over production of glucose, VLDL, CRP and coagulation factors by the fatty liver could contribute to the excess risk of cardiovascular disease associated with the metabolic syndrome and NAFLD. Both of the later conditions also increase the risk of type- 2 diabetes and advanced liver disease. The reason why some deposit fat in the liver where as others do not is poorly understood. Individuals with fatty liver are more likely to have excess abdominal fat and inflammatory changes in adipose tissue [21].

\section{Oxidative stress in Chronic obstructive airway disease (COPD)}

Oxidative stress is now recognized as a major predisposing factor in the pathogenesis of COPD. Existing therapies for COPD are ineffective at halting disease progression, with bronchodilators being the mainstay of pharmacotherapy providing symptomatic relief only. Antioxidant capacity in COPD is substantially reduced as a result of cigarette smoking/or exacerbation, due to the continued production of ROS from endogenous sources.

\section{Conclusion}

Elevated levels of reactive oxygen species and carbonyls are found in COPD and this may be associated with increased inflammation, airway remodeling, auto immunity and corticoid resistance. In addition systemic oxidative stress may also be a causal link in many COPD comorbidities such as cardio vascular diseases and metabolic syndrome. Local oxidative stress may also promote the development of cancer [22].

\section{Atrial fibrillation}

There is growing evidence that oxidative stress is involved in the pathogenesis of atrial fibrillation. Many known triggers of oxidative stress such as age, diabetes, smoking, inflammation and renin angiotensin two signaling activation are linked with an increased risk of the arrhythmia. Blocking angiotensin two signaling and other drugs with anti-oxidant properties can reduce the incidence of atrial fibrillation. Now, studies in animal models and human tissue have shown directly that atrial fibrillation is associated with increased atrial oxidative stress. We review the evidence for a role of oxidative stress in causing atrial fibrillation and propose a unifying hypothesis that multiple triggers elicit oxidative stress which acts to enhance the risk of atrial fibrillation through ion channel dysregulation [23].

Atrial fibrillation (AF) may persist due to structural changes in the atria that are promoted by inflammation. C-reactive protein (CRP) a marker of systemic infection predicts cardiovascular events and stroke, a common sequel of AF. Conclusion: CRP is elevated in AF patients. This study is the first to document elevated CRP in non -post operative arrhythmia patients. These findings are reinforced by stepwise CRP elevation s with higher CRP burden. Although the cause of elevated CRP levels in AF patients remains unknown, elevated CRP may reflect an inflammatory state that promotes persistence of $\mathrm{AF}$ [24].

\section{Oxidative stress and vascular disease}

Oxidative stress is associated with several cardiovascular diseases including atherosclerosis, hypertension, congestive heart failure and diabetes. Oxidation of LDL plays a key role in pathogenesis of atherosclerosis.

Oxidative stress plays a key role in endothelial dysfunction associated in these diseases as superoxide $\mathrm{O} 2$ inactivates nitric oxide (NO) and thereby produce endothelial dysfunction. Endothelial dysfunction is associated with and may contribute to an increased risk of cardiovascular events.

\section{Aortic valvular stenosis / Degenerative calcific aortic stenosis}

Non rheumatic tricuspid aortic valve stenosis has many of the morphological characteristics of atherosclerosis including lipid deposition, inflammation and calcification. Risk factors are similar for aortic stenosis, atherosclerosis hypercholestolemia, male gender and smoking, suggesting that pathophysiology of aortic valvular stenosis and atherosclerosis may be similar.

The author postulates that superoxide may play a role in pathophysiology of "degenerative calcific aortic stenosis" [25].

\section{Oxidative stress}

As oxidative stress has been observed in several diseases of macro vascular and micro vascular origin, it will be important to determine its significance in these disease processes.

Oxidative stress is defined as imbalanced redox state in which prooxidants overwhelm anti-oxidant capacity, resulting in increased production of reactive oxygen species (ROS).

oxLDL plays a central pathogenic role in the arterial wall. Various known risk factors for atherosclerosis lead to a state of oxidative stress characterized by excessive production of ROS that is not neutralized by the body's antioxidant defense. Kita et al. have shown the athero protective effects of the antioxidant probucol in Watanabe Heritable Hyperlipidemic (WHHL) rabbits as it limited oxidation of LDL and prevented foam cell formation. This work established the significance of ox LDL in atherosclerosis. Now it is well known that oxLDL induced pro -oxidant state is present in all stages of atherosclerosis from the beginning to the acute thrombotic event. ox LDL inhibits the expression of the constitutive endothelial enzyme nitric oxide synthase (eNOS), which inhibits a number of steps involved in atherogenesis. In addition it results in generation of ROS from endothelial cells, vascular smooth muscle cells and macrophages. Release of ROS also includes expression of adhesion molecules on endothelial cells, proliferation of macrophages, stimulates collagen formation in fibroblasts, leads to proliferation and migration of vascular smooth muscle cells and activation of platelets [26]. 
Citation: Zafar R (2015) An Insight into Pathogenesis of Cardiovascular Diseases. J Cardiovasc Dis Diagn 3: 197. doi: $10.4172 / 2329-9517.1000197$

Page 7 of 7

\section{Conclusion}

Diseases of macro vascular and micro vascular circulation have strong association with chronic psychosocial stress in their initial presentation and recurrent episodes. There is increased sympathetic activity and elevated levels of serum cortisol supporting activation of HPA and SAM axes, resulting in increased inflammatory markers such as CRP, interleukin -6 , tumor necrosis factor alpha consistent with systemic inflammation and dyslipidemias by mobilization of free fatty acids. Liver responds by producing LDL-C which are poured in the circulation and reach arteries through inflamed endothelium, causing ox LDL formation, oxidative stress, atherosclerosis and tissue damage in territories of macro vascular and micro vascular circulation. As these conditions share several common features and present as multiple co morbidities in an individual, it supports the hypotheses that these disorders manifest in parallel, having a common pathophysiological mechanism, which is chronic psychosocial stress. Targeting micro vascular circulation disorders by lowering LDL-C may be considered for primary and secondary prevention.

\section{References}

1. Pani L, Porcella A, Gessa GL (2000) The role of stress in the pathophysiology of the dopaminergic system. Mol Psychiatry 5: 14-21.

2. G S Everly, Lating JM (2002) The Anatomy and Physiology of the human Stress Response. A Clinical Guide to the Treatment of the Human Stress Response: 17-51

3. McEwen BS (2008) Central effects of stress hormones in health and disease: Understanding the protective and damaging effects of stress and stress mediators. Eur J Pharmacol 583: 174-185.

4. Padgett DA, Glaser R (2003) How stress influences the Immune Response. Trends Immunol 24: 444-448.

5. McEwen BS (2004) Protection and damage from acute and chronic stress: allostasis and allostatic overload and relevance to the pathophysiology of psychiatric disorders. Ann N Y Acad Sci 1032: 1-7.

6. Pace TW, Mletzko TC, Alagbe O, Musselman DL, Nemeroff CB, et al. (2006) Increased stress-induced inflammatory responses in male patients with major depression and increased early life stress. Am J Psychiatry 163: 1630-1633.

7. Black PH (2002) Stress and the inflammatory of neurogenic inflammation : A review. Brain, Behavior and Immunity 16: 622-653.

8. Erling Falk E (2006) Pathogenesis of Atherosclerosis. J Am Coll Cardiol 47: c7-c12.

9. Libby P, Ridker PM, Hansson GK (2009) Inflammation in Atherosclerosis: From pathophysiology to practice. J Am Coll Cardiol 54: 2129-2138.
10. Pepys MB, Hirschfield GM (2003) C-reactive protein: a critical update. J Clin Invest 111: 1805-1812.

11. Granger DN, Senchenkova E (2010) Inflammation and the Microcirculation. San Rafael (CA): Morgan \& Claypool Life Sciences.

12. Rodrigo R, González J, Paoletto F (2011) The role of oxidative stress in the pathophysiology of hypertension. Hypertens Res 34: 431-440.

13. Creager MA, Lüscher TF, Cosentino F, Beckman JA (2003) Diabetes and vascular disease: pathophysiology, clinical consequences, and medical therapy: Part I. Circulation 108: 1527-1532.

14. Holt RI (2004) Diagnosis, epidemiology and pathogenesis of diabetesmellitus : an update for psychiatrists. B J Psych British journal of Psychiatry 184 : 555-563.

15. Seals DR, Kaplon RE, Gioscia-Ryan RA, LaRocca TJ (2014) You're only as old as your arteries: translational strategies for preserving vascular endothelial function with aging. Physiology (Bethesda) 29: 250-264.

16. Skalicky J, Muzakova V, Kandar R, Meloun M, Rousar T, et al. (2008) Evaluation of oxidative stress and inflammation in obese adults with metabolic syndrome. Clin Chem Lab Med 46: 499-505.

17. Neumann J, Ligtenberg G, Klein II, Koomans HA, Blankestijn PJ (2004) Sympathetic hyperactivity in chronic kidney disease: pathogenesis, clinical relevance, and treatment. Kidney Int 65: 1568-1576.

18. Vaziri ND (2004) Role of oxidative stress and antioxidant therapy in chronic kidney disease and hypertension. Curr Opin Nephrol Hypertens 13: 93-99.

19. THAKORE JH (2005) Metabolic Syndrome and Schizophrenia. B J Psych 186.

20. Miller GE, Stetler CA, Carney RM, Freedland KE, Banks WA (2002) Clinical depression and inflammatory risk markers for coronary heart disease. Am J Cardiol 90: 1279-1283.

21. Kotronen A, Yki-Järvinen H (2007) Fatty Liver: A novel component of the metabolic syndrome. Arteriosclerosis, Thrombosis, and Vascular biology 28: 27-38.

22. Kirkham PA, Barnes PJ (2013) Oxidative stress in COPD. Chest 144: 266-273.

23. Iravanian S, Dudley SC. Oxidative Stress and the pathogenesis of Atrial Fibrillation. Current Cardiology 2: 247-254.

24. Chung MK, Martin DO, Sprecher D, Wazni O, Kanderian A, et al. (2001) $\mathrm{C}$-reactive protein elevation in patients with atrial arrhythmias: inflammatory mechanisms and persistence of atrial fibrillation. Circulation 104: 2886-2891.

25. Heistad DD (2006) Oxidative stress and vascular disease: 2005 Duff lecture. Arterioscler Thromb Vasc Biol 26: 689-695.

26. Goyal T, Mitra S, Khaidakov M, Wang X, Singla S, et al. (2012) Current Concepts of the Role of Oxidized LDL Receptors in Atherosclerosis. Curr Atheroscler Rep. 